\title{
COMPARISON OF EFFICACY AND SAFETY OF ULTRASONOGRAPHY AND FIBER OPTIC BRONCHOSCOPE GUIDED PERCUTANEOUS DILATATIONAL TRACHEOSTOMY - A PROSPECTIVE OBSERVATIONAL STUDY Running Title: Efficacy and safety of USG and FOB guided tracheostomy
}

\author{
Nadeem Abu ${ }^{1}$, Chakma Avishek ${ }^{1}$, Siddiqui Obaid Ahmed ${ }^{1}$, Naseem Sana ${ }^{1}$, Hasan Muazzam ${ }^{1 *}$ \\ ${ }^{1}$ Department of Anesthesiology and Critical Care, Jawaharlal Nehru Medical College and Hospital, AMU, Aligarh, Uttar Pradesh
}

Submitted March 4, 2021, Revision received April 22, 2021, Accepted April 23, 2021

\begin{abstract}
Summary
Introduction: We aimed to compare the procedural time, efficacy, and incidence of complications of ultrasonography and fiberoptic bronchoscope-guided percutaneous dilatational tracheostomy (PDT). Methods: The study population included the patients admitted to the department of anesthesiology and critical care. In this prospective observational study, we randomly divided 60 participants into two groups. In the ultrasound (US)-guided group, ultrasonography was used for PDT. While in the FOB guided group, we used the fiberoptic bronchoscope (FOB). We compared efficacy parameters and complications between the two groups. The mean values were compared between study groups using an independent sample t-test. Categorical outcomes were compared using the chi-square test. P-value $<0.05$ was considered statistically significant. Results: Compared with the bronchoscopy group, the US group had a significantly shorter PDT operation time (11.8 \pm 2.5 versus $15.43 \pm 3.27$ mins, $P<0.001)$. FOB group had fewer puncture attempts than the US group. FOB group had more central punctures than the US group $(p<0.001)$. Among PDT complications, bleeding happened in two patients (6.67\%) in the FOB group and tracheal tube cuff puncture was observed in 8 (26.67\%) patients in the US group. Conclusion: The US-guided PDT consumes less time for the procedure compared to bronchoscope-guided PDT. FOB was more effective in terms of parameters like the number of needle puncture attempts, the accuracy of the puncture site, the incidence of the posterior tracheal wall hit compared to US-guided PDT. Complications like bleeding were present in FOB.
\end{abstract}

Keywords: Bleeding; Fiberoptic bronchoscope; Percutaneous dilational tracheotomy; Ultrasonography.

Submitted March 4, 2021, Revision received April 2, 2021, Accepted April 23, 2021

\section{Introduction}

$\mathrm{T}$ racheostomy maintains temporarily or permanently tracheal patency, and it is performed through an incision over the trachea, followed by the insertion of a tracheostomy tube. There are two types of tracheostomies, conventional (surgical) tracheostomy and percutaneous tracheostomy ${ }^{1}$. Percutaneous dilatational tracheostomy (PDT) is routinely performed in the intensive care unit for critically ill patients. In PDT, blunt dissection of pre-tracheal tissues is done, followed by dilatation of trachea over the guidewire and insertion of tracheal cannula using the Seldinger technique ${ }^{2,3}$.

Bronchoscopy guidance has been traditionally used as an adjunct to PDT for safety purposes, as it helps in the appropriate selection of the tracheal puncture site and guides the real-time needle penetration into the trachea, thereby reducing the chance for posterior wall lesions ${ }^{4,5}$. However, bronchoscopy does not distinguish vascular structures or the thyroid gland in the neck region and might cause complications like punctured vessels or a punctured thyroid (local organ lesions). An elevation in intracranial pressure has been reported with bronchoscopy procedures when used in patients with acute brain injury. A higher incidence of complications in obese patients has also been reported, as it is difficult to identify anatomical landmarks on physical examination in such cases ${ }^{6,7}$.

The progress in technical aspects and low invasiveness has increased ultrasound (US) use in 
clinical practice for anesthesia and critical care in recent years ${ }^{8}$. The advantages of US are identification of the cervical vasculature ${ }^{9}$ and more accessible size and length of the tracheal tube selection ${ }^{10}$. Also US helps to identify the most appropriate location of the site for a tracheal puncture and guides the insertion of the needle into the trachea, which is similar to the US-guided vascular puncture technique. The US is helpful in morbid obesity, complex anatomy, and cervical spine problems, which increase the technical difficulty of the procedure. Several authors have recommended pre-procedure cervical US to improve the safety of PDT ${ }^{11,12}$. The mean time needed for the process was $15 \mathrm{~min}$ for FOB-guided PDT and 11 min for US-guided PDT. Some of the complications reported were bleeding, cuff tracheal tube puncture and posterior tracheal wall hit for both procedures ${ }^{13}$.

There is a lack of data comparing the US-guided and bronchoscope-guided percutaneous tracheostomy. Our study hypothesis was that the efficacy and safety of ultrasound-guided percutaneous tracheostomy (US-PDT) using the Griggs technique is superior to fiberoptic bronchoscopy-guided percutaneous tracheostomy (FOB-PDT). The objective of our study is to compare the duration time of fiberoptic bronchoscopy-guided percutaneous tracheostomy (FOB-PDT) and ultrasound-guided percutaneous tracheostomy (US-PDT) using the Griggs technique, and compare the efficacy of both the procedures and the incidence of complications.

\section{Materials and Methods}

\section{Study population and Study site}

The study population included patients admitted to the department of anaesthesiology and critical care, JNMCH, AMU, Aligarh. The inclusion criteria were patients expected to be on prolonged mechanical ventilation $>7$ days, adult patients aged 18-60, and written consent. Exclusion criteria include laryngeal /esophageal/ tracheal carcinoma, soft tissue infection of the neck, anatomical deformity of the neck including enlarged thyroid gland, hematological and terminal malignancies, coagulation disorders, previous tracheostomy, platelet count $<80.000$, INR $>1.4$. This was a prospective observational study. The sample size was calculated assuming the expected mean and standard devia- tion of the total operation time in the ultrasound group as, $\sigma 1(13.5,1)$ and in the bronchoscopy group as, $\sigma 0(14.2,0.9)$, as per the previous study by Jieqiong Song et $\mathrm{al}^{14}$. The other parameters considered for sample size calculation included were $80 \%$ power of the study and 5\% two-sided alpha error. The required sample size was calculated using the following formula as proposed by Kirkwood BR et $\mathrm{al}^{15}$. The formula used for sample size calculation( $\mathrm{N}=$ Sample size; $\mu 1, \mu 0=$ Difference between the means $(\mu 1=13.5$ and $\mu 0=14.2) ; \sigma 1, \sigma 0=$ Standard deviations $(\sigma 1=1$ and $\sigma 0=0.9) ; \mathrm{u}=$ two-sided percentage point of the normal distribution corresponding to $100 \%$; the power $=80 \%, \mathrm{u}=0.84 ; \mathrm{v}=$ percentage point of the normal distribution corresponding to the (two-sided) significance level for significance level $=5 \%, \mathrm{v}=1.960)$. The required sample size as per the calculation mentioned above was 29 in each group. To account for a non-participation rate/loss to follow-up rate of about $3 \%$, another subject will be added to the sample size. Hence, the final required sample size would be 30 subjects in each group. Patients who met the inclusion criteria were randomly (chit in the box technique) divided into two groups, the US-guided group, and the FOB-guided group. We extracted data on all patients admitted to the ICU over 2 years (from September 2018 to September 2020).

The institutional review board and the ethics committee of the hospital approved the study, and informed written consent was taken from the patient's closest relative (Ethics committee number: 243/FM dated 11.5.2019). Data collection included demographics, diagnosis at the time of admission, coagulation profile, e.g., prothrombin time (PT), international ratio (INR) before tracheostomy, and platelet count.

Both the procedures (ultrasound-guided and bronchoscope-guided PDT) were done by surgeon who has 10 years' of experience in the ICU. Ventilatory settings (e.g., tidal volume, respiratory rates, and PEEP) were kept constant, and only $\mathrm{FiO} 2$ increased to $100 \%$. We started sedation before surgery with midazolam $(0.03 \mathrm{mg} / \mathrm{kg})$, analgesia (fentanyl 2 microgram $/ \mathrm{kg}$ ), and vecuronium for complete muscle relaxation $(0.1 \mathrm{mg} / \mathrm{kg})$. The patients were positioned with the head being extended and supported by the assistant; additionally, a small towel roll was placed at the scacj70

pular region to provide the best condition for 
the procedure (extension). Griggs technique was used for percutaneous dilatational tracheostomy ${ }^{16}$.

\section{Ultrasound-guided group procedure}

A single USG device of SonoSite TM and 6-12 $\mathrm{MHz}$ probe was used in all patients in the US group. The incision site was located by a doctor using standard anatomic palpation. Before the procedure, the neck area was examined by USG to identify cricoid cartilage, tracheal rings, blood vessels, and entry point in longitudinal view. Subsequently, a transverse view was used to locate the thyroid gland, vascular structures, trachea, and endotracheal tube. ET tube was withdrawn under vision until the cuff was just below the vocal cords using a C-Mac laryngoscope. A sterile cover was applied over the probe, and a puncture needle filled with normal saline was inserted vertically in out-of-plane mode and advanced until aspiration of air and the needle was observed in the same plane. After aspiration of air and localization of needle, the stylet was removed, and the sheath was left in situ. Manipulation of the ultrasound probe was done by the operator to gain transverse and longitudinal images. A guidewire was inserted through the sheath, which appeared as a hyperechoic shadow on ultrasound.

\section{Fiberoptic Bronchoscope guided group}

A fiberoptic bronchoscope (C-mac: adult size blade, C-mac video laryngoscope by KARL STORTZ, fibreoptic bronschoscope: $5.0 \mathrm{~mm}$ size by STORTZ) was used in this group. The localization of the tracheal space was done by inserting the fiberoptic scope through the catheter mount and withdrawing the scope along with the endotracheal tube until the transillumination was seen over the neck, aspiration of secretion was done as needed, then tracheal rings were identified, and the procedure was performed as described earlier. Procedural complications were recorded during and after the procedure. A third physician independently observed the posterior tracheal wall hit either by the needle or Howard Kelly forceps and needle puncture site through fiberoptic bronchoscope during and after PCT. It is to mention that he had no influence on the procedure.

The timing of the procedure in the USG group was taken from the initial placement of probe to the end marked with tube (tracheal cannula) placement and in the FOB group from the start of insertion of the bronchoscope to the end of the procedure. Details of each tracheostomy procedure collected included time for each procedure, the number of needle puncture attempts, puncture site for the carina, visualization of Griggs forceps, hypotension during the surgery, bleeding, fall in saturation below $90 \%$, the incidence of the posterior tracheal wall hit in each procedure, ET tube cuff puncture and difficult tube placement. A follow-up chest $\mathrm{X}$-ray was done in each patient to look for subcutaneous emphysema. The patient was followed up for five days after the procedure ${ }^{17}$.

Total time taken for the procedure, efficacy (securing the tracheostomy tube with less needle puncture attempt), safety (fewer patients with bleeding and lesser posterior tracheal wall hit), and other complications were considered as primary outcome variables. The study group (ultrasound-guided and fiberoptic bronchoscopy) was considered as the primary explanatory variable. For normally distributed quantitative parameters, the mean values were compared between study groups using an independent sample t-test study group (ultrasound-guided and fiberoptic bronchoscopy). Categorical outcomes were compared between study groups using the chi-square test. P-value $<0.05$ was considered statistically significant. The coGuide version V.1.0 was used for statistical analysis ${ }^{18}$.

\section{Results}

A total of 60 patients were included in the final analysis; 30 (50\%) patients went with the ultrasound-guided, and the remaining 30 (50\%) participants went with fiberoptic bronchoscopy. The mean difference in age between the two groups was statistically not significant $(\mathrm{P}$-value $=0.984$ ). The difference in gender between the study groups was insignificant ( $\mathrm{p}$-value $=0.405)$, with the majority of male participants, 22 ( $73.33 \%)$, being in the ultrasound-guided group. The mean difference of total time taken for the procedure between the two groups was statistically significant ( $\mathrm{p}$-value $<0.001$ ). In an ultrasound-guided group, $5(16.67 \%)$ needle punctures were successful in the first attempt, 15 (50\%) in the 
second attempt, and 10 (33.33\%) participants needed three or more attempts. In the fiberoptic bronchoscopy group, in 23 (76.67\%) of the patients the needle puncture was successful in the first attempt and in 7 (23.33\%) of the patients in the second attempt. In the fiberoptic bronchoscopy group, in all $30(100 \%)$ of the patients visualization of Griggs forceps was possible. In the ultrasound-guided group, in $8(26.67 \%)$ of the patients tracheal cuff was punctured. In the fiberoptic bronchoscopy group, 2 (6.67\%) of the patients had bleeding. In the ultrasound-guided group, in $14(46.67 \%)$ of the patients posterior tracheal wall was hit once, while in the fiberoptic bronchoscopy group, no such occurance has been recorded. (Table 1)

The mean difference of PT, INR, and platelet count between the two groups was statistically insignificant ( $\mathrm{p}$-value $>0.05$ ). Patients were admitted to the critical care unit due to head injury, cerebrovascular accident (CVA), chronic obstructive pulmonary disease (COPD), eclampsia for each, and other diagnoses (Table 2). The difference in the proportion of differential diagnosis between the study groups was statistically insignificant. (P-val$\mathrm{ue}=0.872$ ). (Table 2)

Table 1: Comparison of demographic parameters and procedural outcome between study group. Data are presented as mean \pm SD and $\mathrm{N}(\%)$.

\section{Study group}

\section{Parameter}

Age (in years)

Male

Female

Total time (mins)

Needle puncture

1

2

$>=3$

Central

Not-central

Visualization of Griggs

forceps

Bleeding

Cuff Puncture

Hypotension

$\mathrm{SpO} 2<90 \%$

Subcutaneous emphysema

Difficult tube placement
Ultra Sound-Gu
$38.93 \pm 13.84$

Gender

$22(73.33 \%)$

$8(26.67 \%)$

$11.8 \pm 2.5$

$5(16.67 \%)$

$15(50 \%)$

$10(33.33 \%)$

\section{Puncture site}

\section{Fiber-Optic}

Bronchoscopy $(\mathbf{N}=\mathbf{3 0}) \quad$ P- value

$38.87 \pm 11.16$

0.984*

$$
\begin{aligned}
& 19(63.33 \%) \\
& 11(36.67 \%) \\
& 15.43 \pm 3.27 \\
& 23(76.67 \%) \\
& 7(23.33 \%) \\
& 0(0 \%)
\end{aligned}
$$$$
<0.001 *
$$

$\dagger+$

$24(80 \%)$

$6(20 \%)$

$<0.001 \dagger$

$30(100 \%)$

\section{Complications}

$2(6.67 \%)$

$0(0 \%)$

$0(0 \%)$

$0(0 \%)$

$0(0 \%)$

$0(0 \%)$ $\dagger+$

\section{Posterior tracheal wall hit}

$\begin{array}{lll}\text { None } & 7(23.33 \%) & 29(96.67 \%) \\ 1 \text { time } & 14(46.67 \%) & 1(3.33 \%) \\ 2 \text { times } & 7(23.33 \%) & 0(0 \%) \\ 3 \text { times } & 2 \quad(6.67 \%) & 0(0 \%)\end{array}$

*Independent sample $t$ test, + Chi square test, $\neq$ No statistical test was applied-due to 0 subjects in the cells 
Table 2: Comparison of lab parameter and diagnosis between study group. Data are presented as mean \pm $\mathrm{SD}$ or $\mathrm{N}(\%)$.

\section{Study group}

\begin{tabular}{lllr} 
Parameter & \multicolumn{1}{c}{$\begin{array}{c}\text { USG }(\mathbf{N}=\mathbf{3 0}) \\
\text { Lab parameter }\end{array}$} & FOB $(\mathbf{N}=\mathbf{3 0})$ & P-value \\
PT (s) & $11.9 \pm 1.08$ & $11.79 \pm 1.21$ & $0.729^{*}$ \\
INR & $1.13 \pm 0.13$ & $1.19 \pm 0.13$ & $0.076^{*}$ \\
Platelet count (per microliter) & $170603.33 \pm 52075.84$ & $177873.33 \pm 41762.89$ & $0.553^{*}$ \\
& \multicolumn{1}{c}{ Diagnosis } & & \\
Head injury & $11(36.67 \%)$ & $15(50 \%)$ & \\
CVA & $6(20 \%)$ & $5(16.67 \%)$ & \\
COPD & $3(10 \%)$ & $3(10 \%)$ & $0.872 \dagger$ \\
Eclampsia & $3(10 \%)$ & $2(6.67 \%)$ & $5(16.67 \%)$ \\
Others & $7(23.33 \%)$ & &
\end{tabular}

* Independent sample t- test, $\dagger$ Chi-square test

\section{Discussion}

A total of 60 subjects were included in the final analysis. The time taken for the US PDT was 11.8 \pm 2.5 minutes compared to FOB $15.43 \pm 3.27$ minutes, which was statistically significant. Similarly, in a study by Song J et al., the US group had a significantly shorter PDT operation time $(13.5 \pm 4.4$ minutes for the US group versus $14.2 \pm 3.8$ minutes for the FOB group) ${ }^{14}$. Gobatto et al. also reported similar findings, with the procedure length being shorter for the US group compared to the FOB group ${ }^{13}$.

Multiple types of research focused on the importance of performing percutaneous dilatational tracheostomy with guidance to minimize possible serious complications ${ }^{19,20}$. On the other hand, several reports on the use of bronchoscopy raised concern about potential unwanted side effects like higher incidence of fall in oxygen saturation. Using US as a guide for performing PDT may be safe and effective if used by skilled ICU physicians 21,22 . For these reasons, a comparison between ultrasound-guided PDT and bronchoscopic-guided PDT is necessary.

Most patients (80\%) who had the central site puncture belonged in the fiberoptic bronchoscopy group. In contrast, Song J et al. stated that US guidance helps in precise positioning of the puncture, reducing the distance from the puncture point to the center of the trachea and decreasing the occurrence of complications due to deviation of the tracheostomy tube ${ }^{14}$. Gobatto et al. reported that the US provides good anatomic landmarks and accurate localization of the puncture point; avoids anterior neck structures and vascular injuries; and can guide the puncture needle, penetrating the trachea via the middle line. These results are contradicting the result of the present study ${ }^{13}$.

Two participants in the FOB group had bleeding, while no such cases were observed in the US group. This might be a consequence of the valuable information that the US provides regarding anatomical structures. Numerous studies highlighted that US examination of the neck allows identification of vascular structures in the anterior trachea and reduces the bleeding risk and thereby the complication rate $e^{13,23}$.

Tracheal cuff puncture was recorded in 8 (26.67\%) patients in the ultrasound-guided group. Also, the number of multiple punctures was higher in the US group compared to FOB-guided group, although not statistically significant. In contrast, Ravi PR et al. reported more tracheal cuff punctures 
in the bronchoscopy group compared to the ultrasound group, which was statistically significant. Also, the number of multiple punctures was high in the bronchoscopy group, unlike this study report. US-PDT was rated as moderately difficult, and this was due to the difficulty in identifying the endotracheal cuff balloon ${ }^{24}$. Posterior tracheal wall hit was observed more in the US group (23 participants) compared to the FOB group (1 case).

FOB-guided PDT was more effective than USG guided PDT with almost similar complication rates. The combination of both techniques might be effective, which should be confirmed by further studies. As the present study is an observational study, confounding variables might have affected the results; and groups were naturally unbalanced. This study involves only a single-center investigation, which can affect external validity. The patients were followed for not more than five days and were not assessed for late complications such as tracheal stenosis, vocal abnormalities, or scar characteristics.

\section{Conclusion}

US-guided PDT consumes less time for the procedure compared to bronchoscope-guided PDT. Bronchoscope-guided PDT was effective in terms of the number of needle puncture attempts, the accuracy of the puncture site, visualization of Griggs forceps, and the incidence of the posterior tracheal wall hit compared to US-guided PDT. Bleeding was the complication evident in bronchoscope-guided PDT, while cuff puncture was the complication observed more in US-guided PDT.

Source(s) of support: The project was self-funded. No external agency funded the project.

\section{Presentation at a meeting: Nil}

Conflicting Interest: The authors declare no conflicts of interest.

Acknowledgments: We acknowledge the technical support in data entry, analysis, and manuscript editing by "Evidencian Research Associates".

\section{References}

1. Mehta C, Mehta Y. Percutaneous tracheostomy. Ann Card Anaesth. 2017; 20(5):S19-25.
2. Raimondi N, Vial MR, Calleja J et al. Evidence-based guidelines for the use of tracheostomy in critically ill patients. J Crit Care. 2017; 38:304-18.

3. Australian, Group NZICSCT. Percutaneous Dilatational Tracheostomy Consensus Statement. In: Anzics. 2010. p. 1-25. Available from: papers2://publication/uuid/F70E0556-962C-45F6-9166-2AC8EC818A94

4. Hinerman R, Alvarez F, Keller CA. The outcome of bedside percutaneous tracheostomy with bronchoscopic guidance. Intensive Care Med. 2000;26:1850-6.

5. Fernandez L, Norwood S, Roettger R et al. Bedside percutaneous tracheostomy with bronchoscopic guidance in critically ill patients. J Bronchol. 1997;4:179.

6. Byhahn C, Lischke V, Meininger D et al. Peri-operative complications during percutaneous tracheostomy in obese patients. Anesthesia. 2005;60:12-5.

7. Aldawood AS, Arabi YM, Haddad S. Safety of percutaneous tracheostomy in obese critically ill patients: A prospective cohort study. Anaesth Intensive Care. 2008;36:69-73.

8. Hatfield A, Bodenham A. Ultrasound: An emerging role in anaesthesia and intensive care. $\mathrm{Br}$ J Anaesth. 1999;83:789-800.

9. Flint AC, Midde R, Rao VA et al. Bedside ultrasound screening for pretracheal vascular structures may minimize the risks of percutaneous dilatational tracheostomy. Neurocrit Care. 2009;11:372-6.

10. Hardee PSGF, Ng SY, Cashman M. Ultrasound imaging in the preoperative estimation of the size of tracheostomy tube required in specialised operations in children. $\mathrm{Br} \mathrm{J}$ Oral Maxillofac Surg. 2003;41:312-6.

11. Singh M, Chin KJ, Chan VWS et al. Use of sonography for airway assessment: An observational study. J Ultrasound Med. 2010;29:79-85.

12. Kollig E, Heydenreich U, Roetman B et al. Ultrasound and bronchoscopic controlled percutaneous tracheostomy on trauma ICU. Injury. 2000;31:663-8.

13. Gobatto ALN, Besen BAMP, Tierno PFGMM et al. Comparison between ultrasound- and bronchoscopy-guided percutaneous dilational tracheostomy in critically ill patients: A retrospective cohort study. J Crit Care. 2015;30:220. e13-220.e17.

14. Song J, Xuan L, Wu W et al. Comparison of percutaneous dilatational tracheostomy guided by ultrasound and bronchoscopy in critically ill obese patients. J Ultrasound Med. 2018;37:1061-9.

15. Sharples LD, Kirkwood BR. Essentials of Medical Statistics. J R Stat Soc Ser A (Statistics Soc). 1989;152:263.

16. Griggs WM, Orthley LIG, Gilligan JE et al. A simple percutaneous tracheostomy technique. Surg Gynecol Obstet. 1990;170:543-5.

17. Romero CM, Cornejo RA, Ruiz MH et al. Fiberoptic bronchoscopy-assisted percutaneous tracheostomy is safe in obese critically ill patients: A prospective and comparative study. J Crit Care. 2009;24:494-500.

18. BDSS Corp. Released 2020. coGuide Statistics software, Version 1.0, India: BDSS corp.

19. McCormick B, Manara AR. Mortality from percutaneous dilatational tracheostomy. A report of three cases. Anaesthesia. 2005;60:490-5. 
20. Van Heurn LWE, Theunissen PHMH, Ramsay G et al. Pathologic changes of the trachea after percutaneous dilatational tracheotomy. Pneumologie. 1997;51:262.

21. Gobatto ALN, Besen BAMP, Tierno PFGMM et al. Ultrasound-guided percutaneous dilational tracheostomy versus bronchoscopy-guided percutaneous dilational tracheostomy in critically ill patients (TRACHUS): a randomized noninferiority controlled trial. Intensive Care Med. 2016;42:342-51.

22. Chacko J, Gagan B, Kumar U et al. Real-time ultrasound guided percutaneous dilatational tracheostomy with and without bronchoscopic control: An observational study. Minerva Anestesiol. 2015;81:166-74.

23. Rajajee V, Fletcher JJ, Rochlen LR et al. Real-time ultrasound-guided percutaneous dilatational tracheostomy: A feasibility study. Crit Care. 2011;15(1).

24. Ravi GCPR, Vijay WCMN. Real time ultrasound-guided percutaneous tracheostomy: Is it a better option than bronchoscopic guided percutaneous tracheostomy? Med J Armed Forces India. 2015;71:158-64. 